\title{
COCRIAÇÃO DE VALOR COM ALUNOS: UMA ANÁLISE DA INFLUÊNCIA SOCIAL E DO ENGAJAMENTO NA DISCIPLINA COMO ANTECEDENTES \\ DE FEEDBACK DO ESTUDANTE
}

\author{
VALUE COCREATION WITH STUDENTS: \\ ANALYZING SOCIAL INFLUENCE AND ENGAGEMENT WITH \\ THE DISCIPLINE AS ANTECEDENTS OF STUDENT FEEDBACK
}

Recebido em: 21/10/2013 - Aprovado em: 13/01/2014 Avaliado pelo sistema double blind review Editora Científica: Manolita Correia Lima

\section{TITO LUCIANO HERMES GRILLO titohgrillo@gmail.com \\ THAYS MARTINS DO NASCIMENTO \\ CLÁUDIO DAMACENA \\ VINÍCIUS SITTONI BRASIL \\ PONTIFÍCIA UNIVERSIDADE CATÓLICA DO RIO GRANDE DO SUL}

\begin{abstract}
RESUMO
É natural que educadores de disciplinas vinculadas à área de gestão analisem e reflitam sobre os fenômenos da sala de aula baseando-se em perspectivas que permeiam essa área de conhecimento. Observa-se que educadores e pesquisadores interessados na temática de serviços têm interpretado a sala de aula como um ambiente de encontro de serviço altamente propício à cocriação de valor. O presente estudo procede com tal interpretação, buscando analisar antecedentes do comportamento proativo de fornecimento de feedback do aluno ao professor a fim de promover a melhoria da experiência nos encontros de aula no ensino superior. O estudo avalia, como antecedente direto de feedback, o engajamento na disciplina. Como antecedentes diretos de engajamento, são avaliados constructos oriundos da Teoria da Influência Social. Os resultados, analisados com técnicas de equações estruturais, apresentam relações positivas e com significância entre os constructos, mostrando que, no contexto de sala de aula, processos sociais afetam o relacionamento do estudante com a disciplina e que esse relacionamento propicia o comportamento de colaboração para a melhoria das aulas. Palavras-chave: feedback; engajamento; influência social; cocriação de valor.
\end{abstract}

\footnotetext{
ABSTRACT

It is only natural that educators of management topics often analyze and speculate on classroom phenomena from the points of view prevalent in the field. One will observe that educators and researchers interested in the subject of services may read the classroom as a service environment that is highly propitious to value cocreation The presentstudy follows through in this line as it aims to analyze antecedents of students' proactive behavior of providing feedback to the professor in order to promote improvement in higher education classroom experiences. Engagement with the discipline is assessed as a direct antecedent of the feedback construct, while constructs derived from the Social Influence Theory are assessed as precursors to cengagement. The results, analyzed using structural equation modeling techniques, show positive significant relations among the constructs, demonstrating that, in the classroom context, social processes affect the relationship between students and the academic discipline, and that such relationships are favorable to the behavior of collaborating toward improving the class.

Keywords: feedback; engagement; social influence; value cocreation.
} 


\section{INTRODUÇÃO}

Com base na perspectiva do marketing de serviços, a sala de aula pode ser interpretada como um ambiente de encontro de serviço no qual fenômenos vinculados à cocriação de valor podem ser observados de forma saliente (BRAMbilla; DAMACENA, 20I2; CHUNG; MClarney, 2000). Particularmente em função do elevado nível de complexidade das interações que ocorrem neste ambiente e do relacionamento que se estabelece entre o provedor que está em linha de frente (o professor) e os consumidores (os alunos), a experiência que decorre no ensino de uma disciplina se caracteriza como um serviço complexo (CROSby; evans; COWLes, 1990). O sucesso na cocriação de valor neste ambiente está substancialmente atrelado à condução da experiência que ocorre nele e à evolução do relacionamento entre os atores envolvidos no decorrer dos encontros. Não obstante, reconhece-se que o professor, como funcionário de linha de frente da instituição de ensino superior neste ambiente, torna-se um ator de forte efeito na efetividade da entrega do serviço por meio de sua interação com os estudantes (BRAMBILLA; DAMACENA, 20I2; EIGLIER; LANGEARD, I996).

Sautter, Gagnon e Mohr (2007) - reconhecidos, em 2005, pelo Conselho pelo Avanço e Apoio da Educação (CASE, do inglês Council for the Advancement and Support of Education), como professores de destaque nos Estados Unidos por suas atuações no ensino superior - explicam que a busca pela constante melhoria nas práticas em sala de aula e pela motivação e envolvimento dos estudantes é um componente de suma importância em suas estratégias de ensino. Visto que o processo do ensino superior e, por assim dizer, da entrega do serviço educacional se dá no decorrer de diversos encontros de serviços (diversas aulas), o professor tem por desafio a constante adaptação e renovação de suas abordagens, bem como buscar intensificar o envolvimento dos estudantes com a disciplina. Assim como se assume para diversos outros tipos de serviço que a colaboração do cliente por meio do fornecimento de feedback subsidia a melhoria contínua e até mesmo a inovação no serviço (YI; GONG, 20I3; BETTENCOURT, 1997), também se assume que, no contexto de sala aula, o feedback do estudante é capaz de contribuir 
consideravelmente para que o professor consiga lidar com o desafio da constante adaptação e renovação nas aulas.

Os recentes debates e estudos apoiados conceitualmente nos princípios da lógica dominante de serviço (SDL, do inglês service-dominant logic), discutida com base nas proposições de Vargo e Lusch (2004), indicam que a participação dos consumidores possui um caráter fundamental para que as tentativas de aprimorar as práticas vinculadas à entrega de serviços ocorram de forma a gerar valor. Tal perspectiva é coerente com pesquisas voltadas especificamente para o contexto de ensino superior, uma vez que estudos anteriores já propuseram que, para a geração de valor baseada na experiência em sala de aula, o estudante deve ser interpretado como um stakeholder da disciplina, um ator que tanto afeta como é afetado pelos resultados do encontro de serviço (CHUNG; MCLARNeY, 2000). Tais estudos reconheceram, também, que a participação dos estudantes deve contemplar o fornecimento de informações úteis para a evolução nas práticas de ensino (OLIVER; SAUTTER, 2005).

No entanto, existe uma série de barreiras capazes de afetar negativamente a qualidade dessas informações e a proatividade no seu fornecimento (OLIVER; SAUTTER, 2005). O próprio papel do professor como avaliador do desempenho dos estudantes em aula, por exemplo, é capaz de representar uma barreira. Yi e Gong (2013) assumem que a cocriação de valor está atrelada a tal nível de envolvimento entre o cliente e o serviço, que ele apresenta o comportamento de, discricionariamente, fornecer feedback ao funcionário que está em linha de frente. Não obstante, Chung e McLarney (2000) também argumentam que, para que se gere valor com base na experiência em aula, deve existir uma relação de "intimidade" entre professor e aluno, por meio da qual os estudantes se sintam motivados em relação à disciplina e compromissados com a evolução das aulas, rompendo, assim, as barreiras que afetam o fornecimento de feedback ao professor.

Reconhece-se, porém, que a motivação para que o estudante se envolva com a disciplina de forma a propiciar a cocriação de valor - e, assim, apresente o comportamento discricionário de fornecimento de feedback-, não é única e simplesmente resultante da abordagem de condução das aulas 
realizada pelo professor, por mais relevante que ela seja (OLIVER; SAUTTER, 2005). Esta motivação também é amplamente atrelada a fenômenos sociais associados aos colegas com quem o estudante compartilha as experiências da disciplina (BRAMBILla; DAMACENA, 20I2).

Alinhando as considerações acima, o estudo aqui apresentado foca-se em antecedentes do comportamento discricionário de colaboração do estudante para melhorias e novas práticas em sala de aula: o feedback do estudante. Especificamente, como seu antecedente direto, é proposto e testado o constructo engajamento (VIVEK; BEATTY; MORGAN, 2OI2). Estudos anteriores favorecem a inferência de que o engajamento do estudante na disciplina implica resultados positivos de efetividade do ensino (BRAMBILla; DAMACENA, 2OI2; SKINNER; KINDERMANN; FURRER, 2009; SIMONS-MORTON; CRUMP, 2003), mas há carência de pesquisas que elucidem sobre os motivadores deste constructo no comportamento do indivíduo (PAYNE; STORBACKA; FROW, 2008). Para intervir em tal lacuna, foi proposto e avaliado o efeito de constructos baseados na Teoria da Influência Social (TIS) como seus antecedentes diretos. O objetivo da pesquisa, portanto, consistiu em avaliar o efeito da influência social sobre o engajamento de estudantes de ensino superior na disciplina, e o efeito desse engajamento sobre o feedback do estudante.

\section{FEEDBACK DO ESTUDANTE}

O feedback do cliente é contemplado na literatura de serviços em temas que tratam de comportamentos discricionários. O conhecimento sobre esse tipo de comportamento deriva de pesquisas focadas no comportamento de colaboradores em organizações. Nessas pesquisas, o comportamento discricionário é descrito como ações dos funcionários que não são definidas direta ou explicitamente pelo sistema formal da organização, mas que beneficiam seu funcionamento (ORGAN, 1988). A transposição do conceito para a perspectiva do comportamento do consumidor, por sua vez, o descreve como ações voluntárias do cliente que não são requisitos fundamentais para que o serviço seja entregue com sucesso, mas que contribuem para o desenvolvimento da organização que o fornece (GROTH, 2005). Em suma, o comportamento discricionário ocorre quando o indíviduo realiza ações que 
estão além de suas funções básicas em determinado contexto e que possuem implicações positivas para seu agente de interação.

A fim de criar uma distinção explícita entre as ações do comportamento discricionário e as ações básicas, alguns autores desenvolveram frames específicos. Entre eles, Borman e Motowidlo (I993) elaboraram o framework de particionamento do desempenho do indivíduo. Neste framework, o comportamento discricionário é classificado como desempenho contextual, contemplando comportamentos voltados para o amparo do ambiente organizacional, social e psicológico de um processo, ao passo que as funções básicas do indivíduo são classificadas como desempenho de tarefa, contemplando aspectos tecnicamente necessários a este processo (MOTOWIDLO; VAN SCOTTER, I994). Outros autores fazem a distinção entre desempenho contextual e desempenho de tarefa referindo-se aos mesmos como comportamento extra-role e comportamento in-role, respectivamente (YI; NATARAAJAN; GONG, 20II; BOVE et al., 2009; GROTH, 2005). Em estudos do consumidor, por sua vez, Yi e Gong (2013) referem-se ao desempenho contextual como cidadania do consumidor e ao desempenho de tarefa como participação do consumidor.

O comportamento discricionário do consumidor, ou comportamento de cidadania, envolve a prática de boca-a-boca positivo, o auxílio a outros clientes no ambiente de serviço e, evidentemente, o feedback, que consiste no fornecimento de dados, informações e ideias que a organização pode utilizar para monitorar a qualidade de seus serviços, melhorá-los e até mesmo desenvolver inovações (GROTH, 2005). Considera-se que ações assim representam manifestações de que ocorre a cocriação de valor entre o cliente e o provedor de serviços (YI; GONG, 20I3).

O feedback é de especial relevância para a evolução de serviços. $\mathrm{Na}$ posição de cliente, o indivíduo possui uma perspectiva fundamental para atestar aspectos que devem ser tidos em consideração para o melhoramento do serviço (BETTENCOURT, I997). Tratando-se do encontro de serviço da sala de aula, o feedback implica o aluno comunicar ao professor quando acredita que existem aspectos referentes à experiência de aula que deveriam ser melhorados, elogiar quando considera a aula de boa qualidade e fazer 
sugestões para o uso ou mesmo o desenvolvimento de novas práticas de ensino. Para que ocorra o feedback, é básico que sejam propiciadas interações oportunas entre quem usufrui do serviço e quem o proporciona. Em determinados casos, o feedback se dá por meio de ações estratégicas da organização, como em uma pesquisa de satisfação (GROTH, 2005). Em sua forma mais proativa, porém, o feedback é realizado na interação com o representante da organização que está na linha de frente do serviço (YI; GONG, 20I3). No caso, esse representante é incorporado no professor.

\section{ENGAJAMENTO DO ESTUDANTE NA DISCIPLINA}

Diversas áreas de conhecimento já fizeram uso do termo "engajamento" a fim de se referir a níveis elevados de intensidade na forma como o indivíduo se envolve em determinadas atividades e/ou com determinados objetos (VIVEK; BEATTY; MORGAN, 20I2; BRODIE et al., 20II). Recentemente, pesquisas e debates na área de marketing têm conferido expressiva atenção para este constructo (HOLLEBEEK, 20I3): o Marketing Science Institute (MSI) destacou a demanda por estudos em torno do engajamento em suas prioridades de pesquisa do período 2010-20I2 e periódicos internacionais (Journal of Service Research e Journal of Services Marketing) dedicaram edições especiais ao tema (VIVEK; BEATTY; MORGAN, 20I2). Conceitualmente, o engajamento refere-se conjuntamente à intensidade comportamental e à qualidade emocional que se manifestam no envolvimento ativo do indivíduo com determinado objeto (REEVE et al., 2004; WELLBORN, I99I; CONNELL, 1990). Brodie et al. (20II) explicam que o engajamento ocorre em experiências e interações entre o sujeito e um objeto focal (por exemplo, uma experiência).

Vivek (2009) avalia o engajamento como um constructo composto por três dimensões: (I) entusiasmo; (2) participação consciente - aqui tratada pelo termo "atenção"; e (3) interação social. Entusiasmo reflete um estado ativo de excitamento e de alto nível de energia direcionada ao objeto focal (so; KING; SPARKs, 20I2). O indivíduo entusiasmado se excita quando alcança realizações vinculadas a este objeto (viveK, 2009; GLASSMAN; MCAFEE, 1990). A participação consciente refere-se ao foco despendido pelo indivíduo à atividade (VIVEK, 2009), mas estudos sugerem que esse foco 
pode ser conferido tanto consciente como inconscientemente (ROTHBARD, 200I). Em função disso, neste artigo, utiliza-se o termo atenção para tratar desta dimensão. Seu efeito se dá ao indivíduo direcionar e sustentar sua atenção a elementos vinculados ao objeto focal (VIVEK, 2009; LIN; GREGOR; EWING, 2008; SCHOLER; HIGGINS, 2009). A interação social, por sua vez, refere-se ao fato de o indivíduo engajado se envolver de forma dinâmica e intensa em oportunidades de se relacionar com outros indivíduos que também interagem com o objeto focal (VIVEK, 2009). O aluno engajado, por exemplo, tenderá a ser participativo em debates sobre a disciplina e trocará informações e pensamentos sobre ela.

Pesquisas sugerem que o engajamento pode levar o indivíduo a, proativamente, direcionar esforços que favoreçam sua relação com o objeto focal (so; KING; SPARKS, 20I2). Não obstante, Macey e Schneider (2008) argumentam que o indivíduo engajado atua de forma a ultrapassar os limites da simples manutenção do status quo de seu papel e propõem que se estude o engajamento vinculado a "comportamentos inovativos, demonstrações de iniciativa, proatividade na busca de oportunidades para contribuir e ir além do que, dentro de quadros referenciais específicos, é tipicamente esperado ou exigido" (p. 15). Portanto, o engajamento mostra estreita relação conceitual com comportamentos de cocriação de valor (YI; GONG, 20I3). Coerentemente, o engajamento já foi debatido como rota para esta cocriação (HOLlebeEK 2OI3; viveK; BeATTY; MORGAN, 20I2). Assim, tornase plausível a inferência da seguinte hipótese de pesquisa $(\mathbf{H I})$ :

Hı: O engajamento na disciplina possui efeito de predição positivo sobre o feedback do estudante.

\section{A INFLUÊNCIA SOCIAL NA SALA DE AULA}

Sob a perspectiva de experiência de serviço, o ambiente de sala de aula comporta um fenômeno de consumo colaborativo, dado o nível de influência do conjunto de atores envolvidos no encontro sobre a própria experiência individual (BRAmbilla; DAMACena, 20I2). Coerentemente, a 
Teoria da Influência Social, proposta por Kelman (1958), possui propriedades capazes de contribuir para a explicação de fenômenos observados neste ambiente.

A tis explica processos de natureza social que influenciam comportamentos e atitudes do indivíduo. Kelman (1958) demonstrou a ocorrência de três modos de influência social: (I) complacência; (2) identificação; e (3) internalização. Pesquisas empíricas têm operacionalizado os três modos, respectivamente, por meio dos constructos (I) norma subjetiva; (2) identidade social; e (3) norma de grupo (BAGOZZI; LEE, 2002). A complacência, ou norma subjetiva, refere-se ao efeito gerado em função da crença do indivíduo de que, se adotar determinada atitude, haverá reações favoráveis do grupo ou de alguém que é relevante para ele (KELMAN, 1958). A identificação, ou identidade social, reflete que, ao adotar determinado comportamento, o indivíduo não almeja unicamente os benefícios diretos daquele comportamento, mas também almeja reforçar sua identificação com o grupo, ou sua autoidentidade, nas esferas cognitiva, afetiva e avaliativa do sentimento de pertencimento gregário (BAGOZZI; LEE, 2002; KELMAN, 1958). Por fim, a internalização, ou norma de grupo, ocorre quando o indivíduo adota determinado comportamento existente em um grupo em função de o comportamento ser coerente com o seu próprio sistema de valores (KELMAN, I958).

Em pesquisas voltadas para contextos de relações colaborativas entre consumidores, verificou-se que a avaliação dos três modos sugere que a influência social possui efeitos positivos sobre comportamentos participativos do indivíduo, como a intenção de continuar realizando determinada atividade e a dedicação que o indivíduo despende a ela (ZHOU, 2OII; CHEUNG; LEE, 2OIO; NAMBISAN; BARON, 2009; BAGOZZI; LEE, 2002). Conceitualmente, So, King e Sparks (2012), Brodie et al. (201I), Van Doorn et al. (2010), Libai et al. (2010) e Vivek (2009), discutindo o constructo engajamento, apresentam argumentos para que, entre os estímulos contextuais que levam o indivíduo a se engajar, aspectos da esfera social da experiência de serviço sejam considerados merecedores de destacável atenção.

O processo de influência social caracterizado como complacência, a norma subjetiva, envolve a expectativa que o indivíduo possui quanto 
às respostas a um determinado comportamento. Pode-se considerar, por exemplo, que parte do engajamento do estudante na disciplina é efeito de sua expectativa de reações que seus pais, seus amigos ou mesmo o próprio professor podem apresentar como resposta ao seu envolvimento e consequente desempenho na disciplina. Nesse sentido, o engajamento pode ser avaliado como resultante de um processo mental deliberativo (DULANY, 1997), estimulado pela complacência. Este processo mental significa que o indivíduo reflete e avalia os possíveis efeitos de suas ações. O indivíduo realiza uma reflexão considerando seus desejos, transformando-os em motivação (FRANKFURT, 1988). Se for suposto que obter determinada reação por parte dos pais, de amigos ou do professor pode se estabelecer como um desejo, a norma subjetiva pode atuar como fator a contribuir para o engajamento na disciplina. Torna-se, portanto, plausível a inferência delimitada na seguinte hipótese de pesquisa $(\mathbf{H 2})$ :

H2: A norma subjetiva, no contexto de sala de aula, possui efeito de predição positivo sobre o engajamento na disciplina;

O processo de identificação, a identidade social, está relacionado a comportamentos que buscam estabelecer uma espécie de favoritismo no contexto de grupo (elLemers; KorTeKaAs; OUWERKERK, 1999). Isto é: a influência social que se dá por meio da identificação leva o indivíduo a buscar a autoafirmação de sua identidade expressa na forma de reconhecimento dele pelos outros integrantes do grupo. Não obstante, em análise no contexto organizacional, verificou-se que a identidade social possui efeito positivo em esforços discricionários da parte de funcionários como forma de destacar-se (BERGAMI; BAGOZZI, 2000). Ademais, alinhando as perspectivas de Tajfel e Turner (1986) e de Crocker et al. (1994), o processo de identificação promove comportamentos que levam ao reforço da autoestima, tanto em nível pessoal como coletivo. Assim, no contexto de sala de aula, este processo pode levar o estudante a intensificar sua relação com um objeto focal que seja comum a ele e ao grupo - que, no caso, consiste na disciplina -, a fim de buscar a autoafirmação com aquele grupo por meio do seu 
reconhecimento ou mesmo do favoritismo entre atores do contexto. $\mathrm{O}$ estudante é, portanto, capaz de engajar-se na disciplina por meio de um processo intrínseco que visa à manutenção de sua autodefinição com o grupo, como sugere a afirmação da seguinte hipótese de pesquisa $\left(\mathbf{H}_{3}\right)$ :

H3: A identidade social, no contexto de sala de aula, possui efeito de predição positivo sobre o engajamento na disciplina;

A internalização, ou norma de grupo, reflete um processo social que assume que a intensidade com que o indivíduo se permite envolver em atividades que ocorrem em contextos coletivos/colaborativos é uma função do grau de sua percepção de que compartilha valores e, inextricavelmente, objetivos com os outros indivíduos daquele contexto (BAGOZZI; LEE, 2002). No contexto de sala de aula, uma série de objetivos pode ser percebida como objetivos compartilhados pelo grupo. Por exemplo: obter êxito na realização de um trabalho; obter resultados favoráveis nas avaliações dos professores; ou mesmo elevar o domínio de conhecimento em torno dos tópicos da disciplina. A identificação de congruências de interesses vinculados à disciplina pode se tornar um fator motivacional para o estudante intensificar seu envolvimento com ela. Tal inferência é coerente ao entendimento de que o indivíduo engajado tende a efetivar interações com outros indivíduos que também possuem interesse e dominam conhecimentos vinculados ao objeto focal (WIERTZ; DE RUYTER, 2007; VIVEK, 2009). Em função disso, estabelece-se a seguinte hipótese de pesquisa $\left(\mathbf{H}_{4}\right)$ :

H4: A norma de grupo, no contexto de sala de aula, possui efeito de predição positivo sobre o engajamento na disciplina.

As hipóteses apresentadas nesta seção $\left(\mathbf{H}_{2}, \mathbf{H}_{3}\right.$ e $\left.\mathbf{H}_{4}\right)$, juntamente com a hipótese formulada na seção anterior $(\mathbf{H I})$, configuram o modelo teórico da pesquisa, apresentado na Figura I. 
Figura I Modelo teórico da pesquisa.

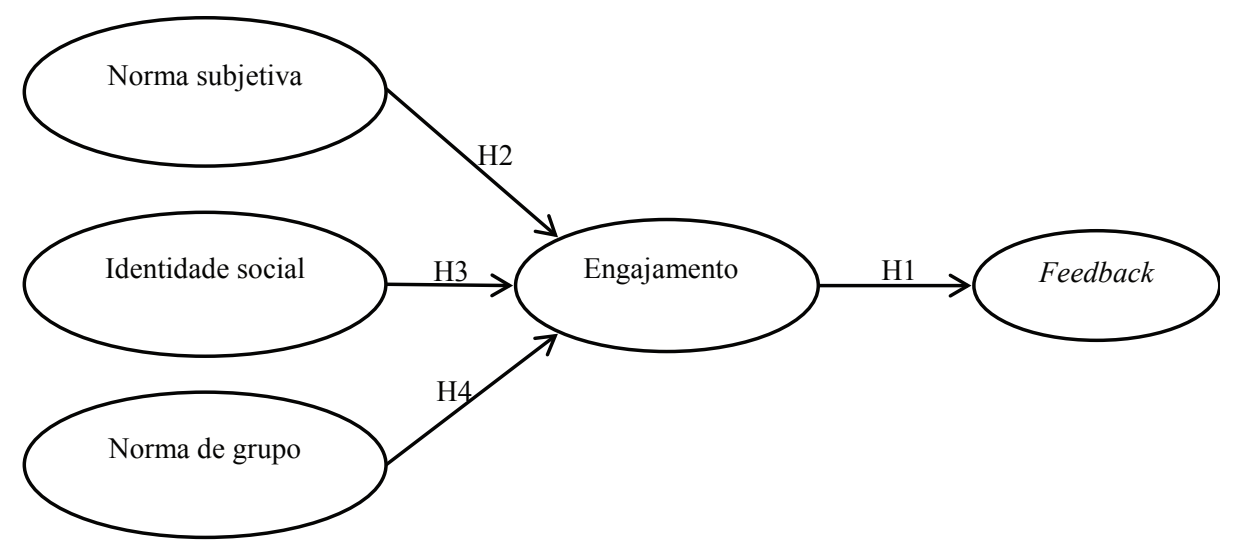

\section{PROCEDIMENTOS METODOLÓGICOS}

Foi conduzida uma survey do tipo corte-transversal. A coleta de dados foi realizada durante o mês de março (2013) em duas universidades privadas localizadas no Rio Grande do Sul, Brasil. A amostra final é composta de 32I estudantes, sendo que 51\% são do sexo masculino, 66\% se inserem na faixa etária de I8 a 23 anos e 6r\% mantêm emprego paralelo aos estudos. Nas subseções que seguem, são descritos (I) o desenvolvimento do instrumento de coleta de dados e (2) a técnica de análise de dados utilizada.

\section{INSTRUMENTO DE COLETA DE DADOS}

O instrumento de coleta de dados foi elaborado no formato de questionário e foram utilizadas escalas existentes e validadas. Para as respostas, adotouse escalas do tipo Likert de sete pontos, sendo que o ponto "I" expressava "discordo totalmente" e o ponto " 7 " expressava "concordo totalmente". Os respondentes foram orientados a utilizar, como referência para responder aos itens, uma disciplina cursada por eles nos últimos doze meses.

Para a mensuração do comportamento discricionário de contribuição para melhorar as práticas em sala de aula foi adaptada a escala do constructo 
feedback de Yi e Gong (2013). Esta consiste em uma escala unidimensional composta por três itens.

O engajamento dos estudantes foi avaliado como um constructo reflexivo de segunda ordem composto pelas dimensões (I) entusiasmo, (2) atenção e (3) interação social. Seguindo o procedimento de So, King e Sparks (2012), a dimensão entusiasmo foi mensurada com três itens da escala de Vivek (2009) juntamente com dois itens gerados por eles em sua pesquisa. Atenção foi mensurada com três itens de Vivek (2009) acrescidos de três itens de Rothbard (200I). Interação social foi avaliada com os quatro itens de Wiertz e De Ruyter (2007).

A influência social em sala de aula foi observada por meio dos constructos (I) norma subjetiva, (2) identidade social e (3) norma de grupo. A escala de norma subjetiva consistiu de dois itens adaptados de Taylor e Todd (I995). Identidade social foi mensurada como um constructo reflexivo de segunda ordem composto por uma dimensão cognitiva, uma dimensão afetiva e uma dimensão avaliativa, sendo que cada dimensão foi composta de três itens adaptados de Zhou (20II). Norma de grupo foi mensurada com dois itens, também adaptados de Zhou (20II).

Todos os itens utilizados no instrumento foram traduzidos para a língua portuguesa e submetidos ao processo de tradução reversa (DILLON; MADDEN; FIRTLE, 1994). Ademais, foi realizada uma simulação do instrumento na qual ele foi aplicado, individualmente, em seis estudantes de graduação. Os estudantes relataram sua interpretação de cada item para que se pudesse avaliar sua clareza e sua validade de face (NUNNALLY; BERNSTEIN, 1994). As versões finais dos itens constam nas Tabelas i e 2 da seção de apresentação dos resultados.

\section{TÉCNICA DE ANÁLISE DE DADOS}

Os dados foram analisados por meio de equações estruturais, com métodos de partial least squares (PLS), conduzidas com auxílio do software SmartPLS 2.o. A adoção de PLs se deu em função de suas propriedades para (I) lidar com amostras inferiores a Io casos por parâmetro do modelo, como seria necessário para o uso de métodos de maximum likelyhood (MARôco, 20IO); (2) lidar com conjuntos de dados que não apresentam normalidade, como é o caso da base 
de dados utilizada neste estudo; e (3) lidar com variáveis latentes formadas por menos de três variáveis observáveis, como é o caso dos constructos norma subjetiva e norma de grupo (HAIR et al., 2OI3; FORNELL; BOOKSTEIN, 1982).

Para a avaliação do outer model (modelo de mensuração), observou-se: (I) as cargas fatoriais; (2) a significância (t) das cargas; (3) a consistência interna do modelo, por meio da confiabilidade composta (CC); (4) a sua validade convergente, por meio da variância média extraída (AVE, do inglês average variance extracted); e (5) a sua validade discriminante, com base no critério Fornell-Larcker. No inner model (modelo estrutural) foram observados: (I) os valores das regressões ( $\beta$ ); (2) a significância das regressões (t); (3) a variância explicada dos constructos endógenos $\left(\mathrm{R}^{2}\right)$; e (4) a relevância de predição $\left(\mathrm{Q}^{2}\right)$. 


\section{APRESENTAÇÃO DOS RESULTADOS}

\section{OUTER MODEL}

As Tabelas i e 2 apresentam os valores obtidos nas cargas fatoriais das variáveis observáveis, juntamente com suas respectivas significâncias $(t)$, e os valores de CC e de AVE das variáveis latentes de primeira ordem. Quanto aos constructos de segunda ordem, as cargas obtidas para as relações entre as dimensões entusiasmo, atenção e interação social com engajamento foram, respectivamente, o,920 ( $\mathrm{t}=\mathrm{II} 7, \mathrm{I3} 3), 0,932(\mathrm{t}=\mathrm{I02}, 690)$ e o,783 $(\mathrm{t}=3 \mathrm{I}, 647)$; e, para as relações entre as dimensões cognitiva, afetiva e avaliativa com o constructo identidade social, foram, respectivamente, o,792 ( $t=31,024), 0,907$ $(\mathrm{t}=88,4 \mathrm{I} 4)$ e $0,833(\mathrm{t}=4 \mathrm{I}, 72 \mathrm{I})$. Também referente aos constructos de segunda ordem, obteve-se $\mathrm{CC}=0,9506$ e $\mathrm{AVE}=0,5652$ para engajamento; e $\mathrm{CC}=0,9250$ e $A V E=0,5803$ para identidade social. Todos os $t$-values obtidos foram superiores a 2,58, o que indica que os coeficientes são todos significantes em nível de $\mathrm{p}<0$,oI (HAIR; RINGLE; SARSTEDT, 20II).

Para atribuir consistência interna ao modelo, os valores de CC devem ser superiores a 0,70. Como visto, todos os constructos atendem a esta prescrição. A validade convergente das medidas do modelo pôde ser atestada por meio dos valores das AVES, que foram unanimemente superiores a 0,50 (valor mínimo aceitável conforme HAIR; RINGLE; SARSTEDT, 20II). Ademais, todas as cargas fatoriais também foram superiores a o,70 e possuem significância, atestando confiabilidade aos indicadores e reforçando a validade convergente. A validade discriminante foi verificada com base no critério Fornell-Larcker, em que a raiz quadrada do valor de AvE da variável latente deve ser superior aos valores de suas correlações com outras variáveis latentes do modelo (FORNELL; LARCKER, 198I). Os resultados desta avaliação, apresentados na Tabela 3, mostram que os constructos do modelo explicam maior nível de variância por meio de suas respectivas variáveis observáveis do que de maneira conjunta com outros constructos, o que indica que há validade discriminante no modelo. 


\section{Tabela I Resultado dos constructos de primeira ordem do outer model}

\section{Feedback $(C C=0,8793 ; \quad A V E=0,7085)$}

FB1: Se eu percebo um problema em relação à aula dada pelo professor, eu falo para ele.' (carga fatorial=0,811; $t=30,444$ )

FB2: Se eu acho que a aula está boa, eu faço comentários sobre isto para o professor.' (carga fatorial $=0,858 ; \mathrm{t}=37,301)$

FB3: Se eu tenho uma ideia útil que pode contribuir para tornar melhor a atividade desta aula, eu comunico ao professor, ou aos colegas da turma/grupo, se for o caso. ${ }^{1}$ (carga fatorial=0,855; $\mathrm{t}=37,877$ )

\section{Engajamento: entusiasmo $(C C=0,9356 ; \quad A V E=0,7445)$}

ENT1: Eu me sinto empolgado em relação às aulas desta disciplina. ${ }^{2}$ (carga fatorial $=0,893 ; t=81,607$ )

ENT2: Eu curto muito as aulas desta disciplina. ${ }^{3}$ (carga fatorial $=0,889 ; \mathrm{t}=56,785$ )

ENT3: Gosto de passar boa parte do meu tempo livre pensando sobre esta disciplina. ${ }^{3}$ (carga fatorial=0,779; $\mathrm{t}=32,842$ )

ENT4: Eu sou entusiasmado em relação às aulas desta disciplina. ${ }^{2}$ (carga fatorial=0,915; $t=91,608$ )

ENT5: Eu sentiria falta das aulas desta disciplina se não estivesse cursando ela. ${ }^{3}$ (carga fatorial $=0,831 ; t=44,229$ )

Engajamento: atenção ( $C C=0,9334 ; \quad A V E=0,7011)$

AT1: Coisas relacionadas a esta disciplina chamam a minha atenção. ${ }^{3}$ (carga fatorial=0,830; $t=39,540$ )

AT2: Eu presto bastante atenção em coisas relacionadas a esta disciplina. ${ }^{3}$ (carga fatorial $=0,872 ; t=60,095$ )

AT3: Eu gosto de saber mais coisas sobre esta disciplina. ${ }^{3}$ (carga fatorial $=0,887 ; t=62,507$ )

AT4: Dedico uma porção significativa de atenção a esta disciplina. ${ }^{4}$ (carga fatorial $=0,832 ; t=36,529$ )

AT5: Eu fico bastante tempo pensando na disciplina. ${ }^{4}$ (carga fatorial $=0,736 ; t=23,698$ )

AT6: Eu me concentro em coisas relacionadas a esta disciplina. ${ }^{4}$ (carga fatorial $=0,859 ; \mathrm{t}=52,095$ )

Engajamento: interação social ( $C C=0,8976 ; \quad A V E=0,6881)$

INT1: De forma geral, eu gosto de me envolver em debates da turma ou do grupo desta disciplina. ${ }^{5}$ (carga fatorial $=0,862 ; \mathrm{t}=63,059$ )

INT2: Eu gosto de interagir com pessoas que pensam como eu nas aulas desta disciplina. ${ }^{5}$ (carga fatorial=0,731; $t=19,476$ )

INT3: Eu gosto de participar ativamente nos debates desta disciplina. ${ }^{5}$ (carga fatorial $=0,903 ; t=82,481$ )

INT4: Em geral, gosto muito de trocar ideias com outras pessoas nesta turma ou no grupo. ${ }^{5}$ (carga fatorial $=0,811 ; \mathrm{t}=31,517$ )

Norma subjetiva $(C C=0,9344 ; \quad A V E=0,8769)$

SN1: Pense em uma ou mais pessoas que você considera importante. Esta(s) pessoa(s) acredita(m) que é bom que você seja um participante nesta turma ou neste grupo. ${ }^{6}$ (carga fatorial $=0,933 ; t=68,487$ )

SN2: Pense em uma ou mais pessoas que influencia $(\mathrm{m})$ o seu comportamento. Esta(s) pessoa(s) acredita(m) que é bom que você seja participativo nesta turma ou neste grupo. ${ }^{6}$ (carga fatorial $=0,940 ; t=91,563$ )

SN2: Pense em uma ou mais pessoas que influencia $(\mathrm{m})$ o seu comportamento. Esta(s) pessoa $(\mathrm{s})$ acredita $(\mathrm{m})$ que é bom que você seja participativo nesta turma ou neste grupo. ${ }^{6}$ (carga fatorial $=0,940 ; \mathrm{t}=91,563$ )

Fonte: Adaptado de ${ }^{1}$ Yi e Gong (2013); ${ }^{2}$ So, King e Sparks (2012); ${ }^{3}$ Vivek (2009); 4Rothbard (2001); 5Wiertz e De Ruyter (2007); 6Taylor e Todd (1995). 
Tabela 2 Resultado dos constructos de primeira ordem do outermodel (continuação) Identidade social: cognitiva $(C C=0,8962 ; \quad A V E=0,7423)$

CSI1: Minha identidade pessoal possui similaridades significativas com a identidade da turma ou do grupo. (carga fatorial $=0,884 ; \mathrm{t}=55,774$ )

CSI2: Minha imagem pessoal possui similaridades significativas com a identidade da turma ou do grupo. (carga fatorial $=0,874 ; \mathrm{t}=47,625$ )

CSI3: Meus valores pessoais possuem similaridades significativas com a identidade da turma ou do grupo. (carga fatorial $=0,826 ; \mathrm{t}=37,742$ )

Identidade social: afetiva $(C C=0,9515 ; \quad A V E=0,8673)$

ASI1: Eu sinto um forte sentimento de pertencimento à turma ou ao grupo. (carga fatorial=0,929; $\mathrm{t}=96,502)$

ASI2: Eu sinto uma forte ligação com a turma ou o grupo. (carga fatorial=0,939; $t=111,283$ )

ASI3: Eu sinto um forte sentimento de ser parte desta turma ou deste grupo. (carga fatorial=0,926; $\mathrm{t}=89,324)$

Identidade social: avaliativa $(C C=0,9286 ; \quad A V E=0,8127)$

ESI1: Eu sou integrante de valor da turma ou do grupo. (carga fatorial=0,886; $t=62,263$ )

ESI2: Eu sou um integrante que exerce influência na turma ou no grupo. (carga fatorial=0,917; $t=89,639$ ) ESI3: Eu sou um integrante importante da turma ou do grupo. (carga fatorial=0,901; $t=67,163$ )

Norma de grupo $(C C=0,8915 ; \quad A V E=0.8043)$

Enunciado: Pense em um objetivo que a turma ou o grupo em que você pensou compartilha (por exemplo, saber o que vai cair na prova ou concluir o trabalho X com notas boas).

GN1: Qual é o nível de intensidade deste objetivo na turma ou no grupo? (carga fatorial $=0,892 ; \mathrm{t}=46,126$ )

GN2: Qual é o nível de intensidade deste objetivo para você? (carga fatorial $=0,902 ; t=48,083$ )

Fonte: Adaptado de Zhou (2011).

Tabela 3 Matriz de correlações

\begin{tabular}{|c|c|c|c|c|c|c|c|c|c|}
\hline Constructo/dimensão & 1 & 2 & 3 & 4 & 5 & 6 & 7 & 8 & 9 \\
\hline 1. Identidade social: afetiva & $0,9313^{1}$ & & & & & & & & \\
\hline 2. Engajamento: atenção & $0,2849^{2}$ & $0,8373^{1}$ & & & & & & & \\
\hline 3. Identidade social: cognitiva & $0,6147^{2}$ & $0,2897^{2}$ & $0,8616^{1}$ & & & & & & \\
\hline 4. Entusiasmo & $0,2976^{2}$ & $0,7991^{2}$ & $0,2443^{2}$ & $0,8628^{1}$ & & & & & \\
\hline 5. Identidade social: avaliativa & $0,6438^{2}$ & $0,3264^{2}$ & $0,4541^{2}$ & $0,3649^{2}$ & $0,9015^{1}$ & & & & \\
\hline 6. Feedback & $0,2415^{2}$ & $0,4150^{2}$ & $0,1849^{2}$ & $0,4569^{2}$ & $0,3919^{2}$ & $0,8417^{1}$ & & & \\
\hline 7. Norma de grupo & $0,3159^{2}$ & $0,3635^{2}$ & $0,2833^{2}$ & $0,3704^{2}$ & $0,3162^{2}$ & $0,1803^{2}$ & $0,8968^{1}$ & & \\
\hline $\begin{array}{l}\text { 8. Engajamento: interação } \\
\text { social }\end{array}$ & $0,4280^{2}$ & $0,6021^{2}$ & $0,3278^{2}$ & $0,5955^{2}$ & $0,5072^{2}$ & $0,5123^{2}$ & $0,3542^{2}$ & $0,8295^{1}$ & \\
\hline 9. Norma subjetiva & $0,4357^{2}$ & $0,3660^{2}$ & $0,3815^{2}$ & $0,3448^{2}$ & $0,5063^{2}$ & $0,3540^{2}$ & $0,3843^{2}$ & $0,4534^{2}$ & $0,9364^{1}$ \\
\hline
\end{tabular}

Notas: ${ }^{1}$ raiz quadrada da AVE; ${ }^{2}$ correlações das variáveis latentes. 


\section{INNER MODEL}

A Figura 2 apresenta os resultados obtidos nas regressões do inner model. Novamente, todos os $t$-values mostram que os valores obtidos para $\beta$ são significante em nível de $\mathrm{p}<0$,or. Assim, a Hi foi suportada, visto que o efeito preditivo de engajamento sobre feedback $(\beta=0,5$ Io) foi significante e $26 \%$ da variância de feedback foi explicada por esta relação. Ademais, utilizando a técnica de blindfolding (WOLD, 1982), conduziu-se o teste da relevância de predição. Valores de $\mathrm{Q}^{2}$ superiores a o mostram que a variável exógena possui poder preditivo relevante sobre sua variável endógena. No caso, a variável endógena feedback apresentou $\mathrm{Q}^{2}=0,182$, indicando relevância de predição para a variável exógena engajamento.

Verificou-se, também, que a $\mathbf{H}_{2}$, a $\mathbf{H}_{3}$ e a $\mathbf{H}_{4}$ possuem suporte, visto que os valores de $\beta$ possuem significância $(\mathrm{p}<0$,or) e o modelo explica $29 \%$ da variância em engajamento. Observou-se que, como variável endógena, engajamento apresentou $\mathrm{Q}^{2}=0, \mathrm{I} 6 \mathrm{I}$, indicando uma capacidade de predição relevante para norma subjetiva, identidade social e norma de grupo como variáveis exógenas.

Figura 2 Resultados das regressões do inner model

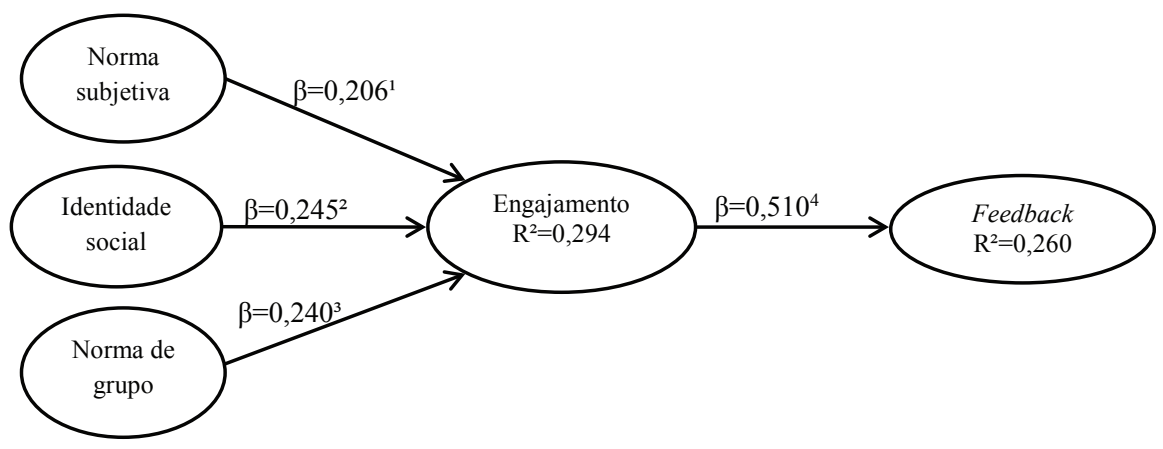

Notas: ${ }^{1} \mathrm{t}=3,199 ;{ }^{2} \mathrm{t}=3,950 ;{ }^{3} \mathrm{t}=4,606 ;{ }^{4} \mathrm{t}=\mathrm{I} 2,843$.

Todavia, é válido ponderar que as magnitudes dos valores de $\beta$ referentes aos efeitos dos constructos da influência social não foram altas. Ao estimar o 
effect size de cada constructo como responsável pela variância explicada em engajamento, obteve-se $\mathrm{f}^{2}=0,042$ (norma subjetiva), $\mathrm{f}^{2}=0,060$ (identidade social) e $\mathrm{f}^{2}=0,062$ (norma de grupo). Portanto, constatou-se que norma subjetiva, identidade social e norma de grupo, embora tenham se mostrado preditores de engajamento, representam variáveis exógenas com baixos efeitos. 
Analisando a sala de aula do ensino superior como ambiente de encontro de serviço (CHUNG; MCLARNEY, 2000), o presente estudo explora relações entre constructos relevantes para a compreensão de como o estudante se engaja na disciplina e contribui com o professor para a melhoria da experiência em sala de aula. Contribui-se, assim, com investigação de potenciais antecedentes do aprimoramento e desenvolvimento de novas práticas em serviços (SZYMANSKI; KROFF; TROY, 2007), particularmente no que se refere ao envolvimento de clientes favorecendo a cocriação de valor (NAMBISAN, 2002); e com a busca por novos conhecimentos em torno do constructo engajamento e seus constructos relacionais (вRODIE et al., 20II; MSI; 20IO). Especificamente, a pesquisa avaliou os efeitos de processos de influência social e do engajamento na disciplina sobre o feedback do estudante (YI; GONG, 20I3). Para isso, elaborou-se e avaliou-se empiricamente o modelo contemplando as hipóteses formuladas em $\mathrm{HI}_{\mathbf{1}} \mathrm{H}_{2}, \mathrm{H}_{3}$ e $\mathbf{H}_{4}$. Por meio de equações estruturais conduzidas com PLS, os efeitos previstos nas hipóteses da pesquisa foram evidenciados empiricamente.

Observou-se que as relações de efeito preditivo de norma subjetiva, identidade social e norma de grupo sobre o engajamento na disciplina são estatisticamente significantes, mas apresentam magnitudes relativamente baixas. É plausível inferir que tal resultado esteja vinculado ao fato de que norma subjetiva, identidade social e norma de grupo representam três processos distintos de influência social, podendo atuar de forma concorrente entre si e em diferentes graus conforme as circunstâncias. Kelman (1958) exemplifica esta propriedade dos processos de influência social da seguinte forma: com base em pesquisas de opinião pública, atitudes de governantes podem ser tomadas sob o efeito de complacência (norma subjetiva), a fim "evitar ostracismo social ou mesmo perseguição" (p. 59); enquanto que a atitude do indivíduo em relação ao governo, por sua vez, pode ser "amplamente baseada em identificação" (identidade social; p. 59), uma vez que a aceitação de algumas crenças ou atitudes políticas pode estar atrelada a uma manutenção da autodefinição. Logo, 
é possível que cada participante da survey conduzida para esta pesquisa seja impactado de forma mais intensa por um dos processos de influência social do que por outros, dispersando, assim, os efeitos da influência social sobre o engajamento entre os constructos norma subjetiva, identidade social, e norma de grupo. De forma não surpreendente, estudos como os de Bagozzi e Dholakia (2002) e Bagozzi e Lee (2002), que avaliaram de forma conjunta os efeitos destes três constructos, também encontraram resultados que apresentaram magnitudes baixas para os valores de $\beta$, porém com significância. Entretanto, os valores de effect size de norma subjetiva, identidade social e norma de grupo também foram relativamente baixos e o $\mathrm{R}^{2}$ obtido para engajamento indica que uma parcela substancial da variância no constructo permaneceu não explicada pelo modelo. Tais resultados podem ser encarados como fortes indícios para a inferência de que existem antecedentes relevantes para o engajamento na disciplina que não são previstos no modelo avaliado neste estudo.

Em suma, os resultados referentes aos efeitos de influência social sobre o engajamento na disciplina indicam que, dadas as magnitudes dos efeitos, são necessários grandes esforços, capazes de gerar amplas variações nos modos de influência social, a fim de se observar variações sutis no engajamento dos estudantes. Em outros termos, o professor que deseja fortalecer o grau do engajamento na disciplina deve questionar se intervenções nas relações de grupo são uma estratégia apropriada, uma vez que gerarão pouca alteração nos níveis engajamento.

O efeito preditivo de engajamento sobre feedback, por sua vez, foi mais expressivo, reforçando os entendimentos de que (I) o indivíduo engajado é propício a, proativamente, apresentar ações contributivas para a melhoria nas práticas em sala de aula (MACEY; SCHNEIDER, 2008); e de que (2) existem relações positivas entre engajamento e comportamentos de cocriação de valor (HOLLEBEEK, 20I3). Contudo, observou-se também que o constructo engajamento explicou uma parcela expressiva da variância no constructo feedback, considerando que foi avaliado como antecedente direto único, mas a maior parte desta variância permaneceu não explicada no modelo, indicando que existem antecedentes não previstos no mesmo gerando 
efeitos subjacentes. Com isto em vista, a prescrição para profissionais envolvidos no encontro de serviço de educação que desejam cocriar valor com os estudantes com base no feedback é que procurem desenvolver estratégias que balizam o engajamento. Algumas sugestões são dadas por Sautter, Gagnon e Mohr (2007).

\section{LIMITAÇÕES}

A capacidade de generalização dos resultados limita-se, em primeira instância, às características da amostra. Esta contempla, principalmente, indivíduos jovens que residem e estudam no Rio Grande do Sul, Brasil. É possível inferir que notáveis variações dos resultados seriam evidenciadas se a amostra fosse predominada por outra faixa etária ou por residentes de outros estados ou países. De forma semelhante à amostra, o contexto do estudo representa um limitador. Embora o contexto do serviço de ensino superior contemple diversos fatores que favorecem a observação empírica da cocriação de valor (BRAMBILLA; DAMACENA, 20I2; CHUNG; MCLARNEY, 2000), ele também apresenta variáveis intervenientes que podem ter afetado o estudo. Por exemplo, a relação subjacentemente hierárquica que se estabelece entre os alunos e o professor em função dos papeis que cada um assume em sala de aula possivelmente inibe efeitos mais expressivos no constructo feedback.

\section{PESQUISAS FUTURAS}

Como ponto inicial, sugere-se que futuras pesquisas a fim de endereçar questões derivadas do presente trabalho foquem-se nas limitações apresentadas anteriormente. Assim, sugere-se que o modelo desenvolvido seja aplicado em outras regiões, onde a cultura local seja notavelmente distinta da cultura da região em que os dados foram coletados. Tais replicações do modelo seriam de considerável interesse em termos acadêmicos, visto que a investigação de um mesmo modelo em diferentes realidades baliza a generalização de resultados e contribui para a elevação da área em questão quanto ao seu status científico (HUBBARD; ARMSTRONG, 1994).

Além do uso de dados coletados em outras regiões, sugere-se a aplicação do modelo em outros contextos de experiências coletivas/colaborativas, 
como comunidades virtuais e espaços de coworking. Se forem evidenciados efeitos com diferenças substanciais dos evidenciados e reportados no presente artigo, suscita-se que se levantem potenciais variáveis moderadoras das relações avaliadas. Dessa forma, pode-se verificar se existem aspectos específicos da experiência de sala de aula no ensino superior que inibem efeitos de maior magnitude entre os constructos da influência social e o engajamento do indivíduo e mesmo entre engajamento e feedback.

Estimula-se, também, a atenção ao constructo feedback. Primeiramente, porque existem outras formas de se avaliar o feedback que não a adotada na presente pesquisa (GROTH, 2005). Mas, além disso, a recente literatura de comportamento do consumidor em torno de comportamentos discricionários aponta uma carência de conhecimento quanto aos outros antecedentes e aos consequentes do feedback (YI; GONG, 20I3). Os próprios resultados apresentados neste artigo reforçam a necessidade de se propor e avaliar outros potenciais antecedentes do constructo, bem como do constructo engajamento.

Éválido, ainda, comentaro enfoque metodológico da pesquisa. Autilização de equações estruturais implica conferir credibilidade ao estudo sob a perspectiva de generalização estatística, mas também acarreta uma análise que não favorece observações indutivas, o que pode resultar em negligência quanto a variáveis relevantes ao fenômeno de interesse. Nesse sentido, futuros estudos em torno das relações entre influência social, engajamento e feedback poderiam tirar proveito de abordagens metodológicas qualitativas, em especial daquelas que favorecem contribuições de natureza indutiva. 


\section{REFERÊNCIAS}

BAGOZZI, R.P.; DHOLAKIA, U.M. Intentional social action in virtual communities. Journal of Interactive Marketing, v. 16, n. 2, p. 2-21, 2002.

BAGOZZI, R.P.; LEE, K.-H. Multiple routes for social influence: The role of compliance, internalization, and social identity. Social Psychology Quarterly, v. 65, n. 3, p. 226-247, 2002.

BERGAMI, M.; BAGOZZI, R. P. Self-categorization, affective commitment and group self-esteem as distinct aspects of social identity in the organization. British Journal of Social Psychology, v. 39, n. 4, p. 555-577, 2000.

BETTENCOURT, L.A. Customer voluntary performance: customers as partners in service delivery. Journal of retailing, v. 73, n. 3, p. 383-406, 1997.

BORMAN, W.C.; MOTOWIDLO, S.J. Expanding the criterion domain to include elements of contextual performance. In: SCHMITT, N.; BORMAN, W. C. Personnel selection in organizations. São Francisco: Jossey-Bass, 1993. p. 71-98.

BOVE, L.L.; PERVAN, S.J.; BEATTY, S.E.; SHIU, E. Service worker role in encouraging customer organizational citizenship behaviors. Journal of Business Research, v. 62, n. 7, p. 698-705, 2009.

BRAMBILLA, F.R.; DAMACENA, C. Cocriação de valor no ensino superior privado: uma análise etnometodológica com alunos de administração de uma universidade do sul do Brasil. Administração: Ensino e Pesquisa, v. 13, n. 3, p. 455-490, 2012.

BRODIE, R.J.; HOLLEBEEK, L.D.; JURIĆ, B.; ILIĆ, A. Customer engagement: conceptual domain, fundamental propositions, and implications for research. Journal of Service Research, v. 14, n. 3, p. 252-271, 2011.

CHEUNG, C.M.K.; LEE, M. K. O. A theoretical model of intentional social action in online social networks. Decision Support Systems, v. 49, n. 1, p. 24-30, 2010.

CHUNG, E.; McLARNEY, C. The classroom as a service encounter: suggestions for value creation. Journal of Management Education, v. 24, n. 4, p. 484-500, 2000.

CONNELL, J.P. Context, self, and action: a motivational analysis of self-esteem processes across the life span. In: CICCHETTI, D.; BEEGHLY, M. The self in transition: from infancy to childhood. Chicago: The University of Chicago Press, 1990. cap. 4, p. 61-97.

CROCKER, J.; LUHTANEN, R.; BLAINE, B.; BROADNAX, S. Collective selfesteem and psychological well-being among White, Black, and Asian college students. Personality and Social Psychology Bulletin, v. 20, n. 5, p. 503-513, 1994.

CROSBY, L.A.; EVANS, K.R.; COWLES, D. Relationship quality in services selling: an interpersonal influence perspective. The journal of marketing, v. 54, n. 3, p. 68-81, 1990.

DILLON, W.R.; MADDEN, T.J.; FIRTLE, N.H. Marketing Research in a Marketing Environment. St. Louis: Times Mirror, 1994. 
DULANY, D.E. Consciousness in the explicit (deliberative) and implicit (evocative). In: COHEN, J.D.; SCHOOLER, J.W. Scientific Approaches to Consciousness. Mahwah: Lawrence Erlbaum Associates, 1997. p. 179-212.

EIGLIER, P.; LANGEARD, E. Servucción: el marketing de servicios. Madrid: McGrawHill, 1996.

ELLEMERS, N.; KORTEKAAS, P.; OUWERKERK, J.W. Self-categorization, commitment to the group and group self-esteem as related but distinct aspects of social identity. European Journal of Social Psychology, v. 29, n. 2-3, p. 371-389, 1999.

FORNELL, C.; LARCKER, D.F. Evaluating structural equation models with unobservable variables and measurement error. Journal of marketing research, v. 18, n. 1, p. 39-50, 1981.

FORNELL, C.; BOOKSTEIN, F.L. Two structural equation models: LISREL and PLS applied to consumer exit-voice theory. Journal of Marketing Research, v. 19, n. 4, p. 440452, 1982.

FRANKFURT, H.G. The importance of what we care about. Cambridge: Cambridge University Press, 1988.

GROTH, M. Customers as good soldiers: examining citizenship behaviors in internet service deliveries. Journal of Management, v. 31, n. 1, p. 7-27, 2005.

GLASSMAN, M.; McAFEE, R.B. Enthusiasm: the missing link in leadership. Advanced Management Journal, v. 55, p. 3-6, 1990.

HAIR, J.F.; HULT, T.; RINGLE, C.; SARSTEDT, M. A Primer on Partial Least Squares Structural Equation Modeling (PLS-SEM). Los Angeles: SAGE Publications, 2013.

HAIR, J.F.; RINGLE, C.M.; SARSTEDT, M. PLS-SEM: Indeed a silver bullet. The Journal of Marketing Theory and Practice, v. 19, n. 2, p. 139-151, 2011.

HOLLEBEEK, L.D. The customer engagement/value interface: an exploratory investigation. Australasian Marketing Journal, v. 21, n.1, p. 17-24, 2013.

HUBBARD, R.; ARMSTRONG, J.S. Replications and extensions in marketing: rarely published but quite contrary. International Journal of Research in Marketing, v. 11, n. 3, p. 233-248, 1994.

KELMAN, H.C. Compliance, identification, and internalization: Three processes of attitude change. Journal of Conflict Resolution, v. 2, n. 1, p. 51-60, 1958.

LIBAI, B.; BOLTON, R.; BUGEL, M.S.; RUYTER, K.; GOTZ, O.; RISSELADA, H.; STEPHEN, A.T. Customer-to-customer interactions: broadening the scope of word of mouth research. Journal of Service Research, v. 13, n. 3, p. 267-282, 2010.

LIN, A.; GREGOR, S.; EWING, M. Developing a scale to measure the enjoyment of web experiences. Journal of Interactive Marketing, v. 22, n. 4, p. 40-57, 2008.

MACEY, W.H.; SCHNEIDER, B. The meaning of employee engagement. Industrial and Organizational Psychology, v. 1, n.1, p. 3-30, 2008. 
MARKETING SCIENCE INSTITUTE. 2010-2012 Research Priorities. Cambridge: Marketing Science Institute, 2010.

MARÔCO, J. Análise de equações estruturais: fundamentos teóricos, software \& aplicações. Pêro Pinheiro: Report Number, 2010.

MOTOWIDLO, S.J.; VAN SCOTTER, J.R. Evidence that task performance should be distinguished from contextual performance. Journal of Applied Psychology, v. 79, n. 4, p. 475-480, 1994.

NAMBISAN, S. Designing virtual customer environments for new product development: toward a theory. Academy of Management Review, v. 27, n. 3, p. 392-413, 2002.

NAMBISAN, S.; BARON, R.A. Virtual customer environments: testing a model of voluntary participation in value co-creation activities. Journal of Product Innovation Management, v. 26, p. 388-406, 2009.

NUNNALLY, J.C.; BERNSTEIN, I.H. Psychometric Theory. 3.ed. Nova York: McGrawHill, 1994.

OLIVER, R.L.; SAUTTER, E. "Pookie". Using course management systems to enhance the value of student evaluations of teaching. Journal of Education for Business, v. 80, n. 4, p. 231-234, 2005.

ORGAN, D. W. Organizational Citizenship Behavior: the good soldier syndrome. Lexington: Lexington Books, 1988.

PAYNE, A.F.; STORBACKA, K.; FROW, P. Managing the co-creation of value. Journal of the Academy of Marketing Science, v. 36, n.1, p. 83-96, 2008.

REEVE, J.; JANG, H.; CARRELL, D.; JEON, S.; BARCH, J. Enhancing students' engagement by increasing teachers' autonomy support. Motivation and Emotion, v. 28, n. 2, p. 147-169, 2004.

ROTHBARD, N.P. Enriching or depleting? The dynamics of engagement in work and family roles. Administrative Science Quarterly, v. 46, n. 4, p. 655-684, 2001.

SCHOLER, A.A.; HIGGINS, E.T. Exploring the complexities of value creation: The role of engagement strength. Journal of Consumer Psychology, v. 19, n. 2, p. 137-143, 2009.

SAUTTER, E. "Pookie”; GAGNON, G.B.; MOHR, J.J. Educators who have made a difference for their students: observations and reflections of three nationally recognized marketing professors. Journal of Marketing Education, v. 29, n.1, p. 85-90, 2007. SIMONS-MORTON, B. G.; CRUMP, A.D. Association of parental involvement and social competence with school adjustment and engagement among sixth graders. Journal of School Health, v. 73, n. 3, p. 121-126, 2003.

SO, K.K.F.; KING, C.; SPARKS, B. Customer Engagement With Tourism Brands: Scale Development and Validation. Journal of Hospitality \& Tourism Research, 2012. 
SKINNER, E.A.; KINDERMANN, T.A.; FURRER, C.J. A motivational perspective on engagement and disaffection conceptualization and assessment of children's behavioral and emotional participation in academic activities in the classroom. Educational and Psychological Measurement, v. 69, n. 3, p. 493-525, 2009.

SZYMANSKI, D.M.; KROFF, M.W.; TROY, L.C. Innovativeness and new product success: insights from the cumulative evidence. Journal of the Academy of Marketing Science, v. 35, n. 1, p.35-52, 2007.

TAJFEL, H.; TURNER. J. The social identity theory of intergroup behavior. In: WORCHEL, S.; AUSTIN, W.G. Psychology of Intergroup Relations. Chicago: Nelson-Hall, 1986. cap. 3, p. 33-47.

TAYLOR, S.; TODD, P.A. Understanding information technology usage: a test of competing models. Information Systems Research, v. 6, n. 2, p. 144-176, 1995.

VAN DOORN, J.; LEMON, K.N.; MITTAL, V.; NASS, S.; PICK, D.; PIRNER, P.; VERHOEF, P.C. Customer engagement behavior: theoretical foundations and research directions. Journal of Service Research, v. 13, n. 3, p.253-266, 2010.

VARGO, S.L.; LUSCH, R.F. Evolving to a new dominant logic for marketing. Journal of Marketing, v. 68, n. 1, p. 1-17, 2004.

VIVEK, S.D. A Scale of Consumer Engagement. 1996. 233 f. Dissertation (Doctor of Philosophy in the Department of Management and Marketing) - The University of Alabama, Tuscaloosa, 2009.

VIVEK, S.D.; BEATTY, S.E.; MORGAN, R.M. Customer engagement: exploring customer relationships beyond purchase. The Journal of Marketing Theory and Practice, $\mathrm{v}$. 20, n. 2, p. 127-145, 2012.

WELLBORN, J.G. Engaged and disaffected action: the conceptualization and measurement of motivation in the academic domain. 1991. Dissertation (Doctor of Philosophy in the Department of Psychology) - University of Rochester, Rochester, 1991.

WIERTZ, C.; DE RUYTER, K. Beyond the call of duty: why customers contribute to firm-hosted commercial online communities. Organization Studies, v. 28, n. 3, p. 347-376, 2007.

WOLD, H. Soft modeling: the basic design and some extensions. In: JÖRESKOG, K.G.; WOLD, H. Systems Under Indirect Observation Part II. Amsterdam: North-Holland, 1982.

YI, Y.; GONG, T. Customer value co-creation behavior: Scale development and validation. Journal of Business Research, v. 66, n.9, p. 1279-1284, 2013.

YI, Y.; NATARAAJAN, R.; GONG, T. Customer participation and citizenship behavioral influences on employee performance, satisfaction, commitment, and turnover intention. Journal of Business Research, v. 64, p. 87-95, 2011.

ZHOU, T. Understanding online community user participation: a social influence perspective. Internet Research, v. 21, n. 1, p. 67-81, 2011. 


\section{DADOS DOS AUTORES}

\section{TITO LUCIANO HERMES GRILLO^ titohgrillo@gmail.com} Doutorando em Administração na UFRGS e Mestre em Administração e Negócios pela PUC/RS

Instituição de vinculação: Pontifícia Universidade Católica do Rio Grande do Sul Porto Alegre/RS - Brasil

Áreas de interesse em pesquisa: Comportamento do Consumidor, Marketing Estratégico e Inovação.

* Av. Ipiranga, 668I, Prédio 50 Partenon Porto Alegre/RS 906r9-900

THAYS MARTINS DO NASCIMENTO thaysdn@gmail.com Graduanda em Administração com ênfase em Marketing pela PUC/RS Instituição de vinculação: Pontifícia Universidade Católica do Rio Grande do Sul Porto Alegre/RS - Brasil

Áreas de interesse em pesquisa: Comportamento do consumidor.

\section{CLÁUDIO DAMACENA damacena.claudio@gmail.com}

Doutor em Administração pela Universidade de Córdoba (Espanha)

Instituição de vinculação: Pontifícia Universidade Católica do Rio Grande do Sul Porto Alegre/RS - Brasil

Áreas de interesse em pesquisa: Marketing Estratégico, Marketing de Serviços e Comportamento do consumidor.

\section{VINÍCIUS SITTONI BRASIL vinicius@pucrs.br}

\section{Doutor em Marketing pela UFRGS}

Instituição de vinculação: Pontifícia Universidade Católica do Rio Grande do Sul Porto Alegre/RS - Brasil

Áreas de interesse em pesquisa: Experiências de Consumo, Marketing de Serviços e Varejo, Cocriação de Valor. 